\title{
INTOXICAÇÃO EXPERIMENTAL POR Kalanchoe Blossfeldiana (CRASSULACEAE) EM CÃES
}

\author{
Leandro Bertoni Cavalcanti Teixeira, ${ }^{1}$ Raimundo Alberto Tostes, ${ }^{2}$ Silvia Franco Andrade, ${ }^{3}$ \\ Michiko Sakate ${ }^{4}$ e Raquel Ferraz Laurenti ${ }^{5}$ \\ 1. Docente da disciplina de Anatomia Patológica Veterinária do Centro Regional Universitário de Espírito Santo do Pìnhal. \\ E-mail: le-teixeiravet@hotmail.com \\ 2. Docente da disciplina de Anatomia Patológica Veterinária do Centro Universitário de Maringá \\ 3. Professora doutora docente da disciplina de Clínica Médica de Pequenos Animais da Universidade do Oeste Paulista (UNOESTE) \\ 4. Professora doutora docente da disciplina de Clínica Médica de Pequenos Animais da FMVZ UNESP-Botucatu \\ 5. Médica veterinária autônoma.
}

\section{RESUMO}

Este estudo visou à avaliação clínica e laboratorial da administração oral de Kalanchoe blossfeldiana a cães. Para isso foram utilizados doze cães, adultos, com idade entre dois a cinco anos, sem predileção de sexo ou raça. Os animais receberam extratos de $K$. blossfeldiana e foram monitorados clínica e laboratorialmente através de hemograma, dosagem sérica de creatinoquinase (CK), alaninoaminotransferase (ALT), fosfatase alcalina (FA) e eletrocardiograma acerca das alterações provocadas pelos princípios tóxicos da planta. A avaliação dos animais não revelou nenhuma alteração clínica e/ou laboratorial, estando os valores das enzimas CK, ALT, FA e hemograma dentro dos valores normais. No exame eletrocardiográfico todos os animais apresentaram normalidade de ritmo (arritmia sinusal), eixo cardíaco e nas medidas das ondas, não sendo evidenciado nenhum indício de necrose ou isquemia cardíaca. Os resultados mostraram que o extrato de K. bloosfeldiana não produz um quadro de intoxicação em cães nas condições em que foi desenvolvido o experimento.

PALAVRAS-CHAVES: Cães, Crassulaceae, intoxicação, Kalanchoe.

\section{ABSTRACT}

\section{EXPERIMENTAL INTOXICATION BY Kalanchoe Blossfeldiana (CRASSULACEAE) IN DOGS}

The purpose of this study was to evaluate the clinical and laboratorial aspects of the oral administration of Kalanchoe blossfeldiana in dogs. Twelve dogs, from two to five years of age, without breed or sex preferences, were used. The animals were inoculated with the $K$. blossfeldiana extract and monitored by complete blood count (CBC), serum biochemical analysis of creatinokinase (CK), alanine aminotransferase (ALT), alkaline phosfatase (FA) and electrocardiographic exam for any cardio-toxic effect from the extract. No significant changes were identified by clinical examination, $\mathrm{CBC}$, blood chemistry or EKG exams, with all results within standard values. The electrocardiographic exam of all the animals presented normal rhythm (sinus arrhythmia), cardiac axle and wave measures, without necrose or cardiac ischemia indication. These results suggested that $K$. blossfeldiana extract do not cause acute intoxication in dogs within the scope of this experiment.

KEYWORDS: Dogs, Crassulaceae, intoxication, Kalanchoe. 


\section{INTRODUÇÃO}

Os relatos de intoxicação por plantas ornamentais envolvendo cães têm sido cada vez mais frequentes, contudo estes quadros ainda não foram detalhadamente descritos.

Os cães podem ter acesso a diferentes plantas tóxicas em seu ambiente, principalmente em jardins, quintais ou sendo trazidas para o interior das residências pelos proprietários, sem o conhecimento do seu potencial tóxico (MILEWSKI \& KHAN, 2006).

Dentre as plantas de jardim e ornamentação tidas como tóxicas para pequenos animais encontramse plantas do gênero Kalanchoe. Referida vulgarmente como folha-da-fortuna, folha-da-costa, sião ou simplesmente Kalanchoe, a $K$. blossfeldiana é muito popular em ambientes domésticos, pelo fato de apresentar uma multiplicidade de formas, cores e tamanhos (HYAKUTAKE et al., 1972), por ser de fácil cultivo e florescer em qualquer época do ano (WAGNER et al., 1985).

A Kalanchoe sp. tem se mostrado tóxica para várias espécies de animais, incluindo ratos, cães, gatos, aves, bovinos e ovinos (ANDERSON et al., 1983; WILLIAMS \& SMITH, 1984; MACKENZIE et al., 1987; MASVINGWE \& MAVENYENGWA, 1997; MILEWSKI \& KHAN, 2006).

As espécies $K$. diagramontiana, $K$. tubiflora e $K$. fedtschenkoi apresentaram alta toxicidade quando testada à dose de $8-12 \mathrm{mg} / \mathrm{kg}$ em aves de duas semanas de idade, ao passo que as espécies $K$. tomentosa e K. blossfeldiana não apresentaram o mesmo efeito tóxico (WILLIAMS \& SMITH, 1984). MASVINGWE \& MAVENYENGWA (1997) relataram um caso de intoxicação natural por $K$. Lanceolata em bovinos a campo, que apresentaram sinais de intoxicação aguda por glicosídeos cardíacos. ANDERSON et al. (1983) produziram um quadro de intoxicação aguda em ovinos utilizando extrato de $K$. lanceolata Forsk administrada por via oral e intravenosa, em que os animais também apresentaram sinais de intoxicação aguda por glicosídeos cardíacos e lesões histopatológicas que apontaram cardiomiopatia multifocal severa.

A intoxicação por Kalanchoe sp. aparece entre os sete principais tipos de intoxicação reportados, em cães e gatos, ao Centro de Controle de Intoxicação Animal (APCC) de Illinois (Estados Unidos da América), que recebe informações de casos de intoxicação animal do país todo (MILEWSKI \& KHAN, 2006).

Os princípios tóxicos de Kalanchoe sp. variam amplamente. Os glicosídeos cardíacos, entre eles os Bufadienolídeos, são os principais compostos encontrados nas várias espécies de Kalanchoe (STEYN \& HEERDEN, 1998). O bufanolídeo hellibrigenina 3-acetato foi isolado da $K$. lanceolata (ANDERSON et al., 1983; MASVINGWE \& MAVENYENGWA, 1997); a daigremontianina é o principal princípio ativo tóxico de K. daigremontiana (WAGNER et al., 1985) e os flavonoides glicosídeos e componentes fenólicos foram isolados de $K$. pinnata (GAIND \& GUPTA, 1971; GAIND \& GUPTA,1973). Os princípios tóxicos da $K$. tubiflorum são a bryotoxin A 14, bryotoxin B 15 e bryotoxin C 16 (STEYN \& HEERDEN, 1998). Outros componentes fitoquímicos isolados incluem $\alpha$-amirina, $\beta$-amirina, briofilina, glutinol, quercetina, quercetina-3-diarabinosídeo, taraxerol e 3,8-dimetoxi-4',5,7-trihidroxiflavone (ASHTON \& POLY, 1977; MCKENZIE \& DUNSTER, 1986; VARMA et al., 1986; MOURÃO et al., 1999). Como principal efeito tóxico, os glicosídeos cardíacos apresentam a capacidade de aumentar a força de contratilidade da musculatura cardíaca por inibição da enzima $\mathrm{Na}^{+}, \mathrm{K}^{+}$ ATPase, levando à retenção de sódio e cálcio nos miócitos e expulsão de potássio desses, podendo, assim, induzir lesões cardíacas (STEYN \& HEERDEN, 1998; MILEWSKI \& KHAN, 2006).

Os princípios tóxicos se localizam em todas as partes da planta, concentrando-se principalmente nas flores (MILEWSKI \& KHAN, 2006).

Algumas espécies como a $K$. brasiliensis também têm sido estudadas do ponto de vista terapêutico, como agente anti-inflamatório e imunomodulador (MOURÃO et al., 1999; IBRAHIM et al., 2002) e antiulcerativo (PEREZ, et al., 1999). Outras apresentam efeito cicatrizante (LIPETZ, 1967). Ainda a K. pinnata demonstrou ação antimicrobiana, antimutagênica, adjuvante no combate a Leishmania e depressiva do sistema nervoso central (DA SILVA et al., 1995; DA SILVA et al., 1999; UMBUZEIRO-VALENT et al., 1999; SALAHDEEN \& YEMITAN, 2006).

As principais alterações clínicas observadas na intoxicação por Kalanchoe sp. são depressão, incoordenação motora, opistótono, tremores, convulsões e paralisia, seguidos de coma e óbito (ANDERSON et 
al., 1983; WILLIAMS \& SMITH, 1984; MASVINGWE \& MAVENYENGWA, 1997).

Com o presente estudo, objetivou-se avaliar o quadro clínico e laboratorial da intoxicação aguda por extrato obtido de Kalachoe blossfeldiana.

\section{MATERIAL E MÉTODOS}

O estudo foi desenvolvido no Hospital Veterinário da Universidade do Oeste Paulista (UNOESTE), Presidente Prudente, São Paulo.

Foram utilizados doze animais, sete machos e cinco fêmeas, com idade entre um e seis anos, estabelecida pelas características da dentição, de porte médio, pesando entre 7 e $14 \mathrm{~kg}$ de peso vivo e bom estado geral, segundo os parâmetros de MCCURNIN \& POFFENBARGER (1991).

Os cães, procedentes do Canil do Biotério da UNOESTE, foram mantidos em jaulas individuais no Hospital Veterinário, recebendo água e ração ad libitum até 24 horas antes do experimento. Após a inoculação da solução aquosa, os animais foram mantidos em jaulas individuais e acompanhados continuamente por um período de até 24 horas.

Mantiveram-se os animais no Canil Central da UNOESTE, sendo os exames laboratoriais realizados no Laboratório Clínico do Hospital Veterinário da UNOESTE.

Foram aferidos parâmetros clínicos dos animais (frequência cardíaca, temperatura, pulso, tempo de preenchimento capilar e frequência respiratória) antes e até doze horas após a sua intoxicação, com intervalos de duas horas.

Realizou-se a identificação botânica de Kalanchoe blossfeldinana no Laboratório de Botânica da Faculdade de Agronomia da UNOESTE. As mudas foram adquiridas no município de Holambra, SP, e mantidas em ambiente natural até a realização dos inóculos.

As folhas, caule e flores de Kalanchoe blossfeldiana foram cortadas e trituradas, perfazendo um total de duzentas gramas e homogeneizadas com $20 \mathrm{~mL}$ de água destilada.

Esta solução foi então filtrada, utilizando-se papel filtro, sendo administrados $40 \mathrm{~mL}$ do filtrado pela via oral com a ajuda de uma seringa de $20,0 \mathrm{~mL}$.
Também se administrou, por via oral, a parte sólida da solução que se depositou no filtro.

Colheram-se amostras de sangue para os seguintes exames laboratoriais: hemograma e bioquímica sérica, para mensuração das enzimas creatinoquinase (CK), alanina amino transferase (ALT), fosfatase alcalina (FA). Desenvolveram-se exames eletrocardiográficos para a verificação de possíveis alterações cardíacas.

Foram utilizados quatro momentos para a realização de todos os exames: Momento 0, uma hora antes da intoxicação, Momento 1, seis horas após a intoxicação, Momento 2, doze horas após a intoxicação e momento 3, vinte e quatro horas após a intoxicação.

Para comparar as médias de parâmetros séricos bioquímicos, parâmetros hematológicos, parâmetros clínicos e parâmetros eletrocardiográficos foi empregado o teste de análise de variância para amostras pareadas, sendo os contrastes realizados pelo método de Tukey. Para comparar os escores obtidos para os diferentes eixos cardíacos observados nos cães estudados, empregou-se o teste não paramétrico de Friedman. Para ambos os testes, considerou-se o nível de significância de $5 \%(\alpha=0,05)$.

\section{RESULTADOS}

Dos doze animais que receberam o extrato de Kalanchoe blossfeldiana, nenhum morreu e optou-se por não sacrificar nenhum animal para a execução de necrópsia. A avaliação clínica dos animais não revelou nenhuma alteração dos parâmetros clínicos no decorrer dos momentos de avaliação (Tabela 1). Quanto aos exames laboratorias, nenhum dos animais apresentou alterações significativas tanto nos exames bioquímicos quanto no hemograma, estando os valores das enzimas CK, ALT e FA e os valores hematimétricos dentro dos valores normais (Tabelas 2 e 3 ).

No exame eletrocardiográfico, todos os animais apresentaram normalidade de ritmo (arritmia sinusal), eixo cardíaco e nas medidas das ondas. Também não apresentaram nenhum indício eletrocardiográfico de necrose ou isquemia cardíaca (Tabela 4). 
TABELA 1. Médias e desvios-padrão dos parâmetros clínicos, avaliados após uma hora (Momento 0), após seis horas (Momento 1), após doze horas (Momento 2) e após vinte e quatro horas (Momento 3) da administração por via oral de extrato de Kalanchoe blossfeldiana aos cães

\begin{tabular}{ccccc}
\hline \multirow{2}{*}{ Momento } & \multicolumn{4}{c}{ Parâmetro } \\
\cline { 2 - 5 } & $\begin{array}{c}\text { TPC } \\
(\text { segundos })\end{array}$ & $\begin{array}{c}\text { FC } \\
\text { (batimentos/min. })\end{array}$ & $\begin{array}{c}\text { FR } \\
(\text { movimentos } / \text { min })\end{array}$ & t $^{\circ} \mathrm{C}$ \\
\hline 0 & $2.41^{\mathrm{a}} \pm 0.51$ & $112.25^{\mathrm{a}} \pm 10.199$ & $49.66^{\mathrm{ab}} \pm 18.34$ & $38.61^{\mathrm{a}} \pm 0.70$ \\
1 & $2.41^{\mathrm{a}} \pm 0.51$ & $108.83^{\mathrm{a}} \pm 11.101$ & $54.66^{\mathrm{a}} \pm 22.24$ & $38.37^{\mathrm{a}} \pm 0.71$ \\
2 & $2.25^{\mathrm{a}} \pm 0.45$ & $110.83^{\mathrm{a}} \pm 7.602$ & $46.66^{\mathrm{ab}} \pm 4.92$ & $38.30^{\mathrm{a}} \pm 0.74$ \\
3 & $2.41^{\mathrm{a}} \pm 0.51$ & $117.33^{\mathrm{a}} \pm 5.193$ & $48.41^{\mathrm{b}} \pm 7.52$ & $38.51^{\mathrm{a}} \pm 0.62$ \\
\hline
\end{tabular}

$\mathrm{TPC}=$ tempo de preenchimento capilar, $\mathrm{FC}=$ frequência cardíaca, $\mathrm{FR}$ - frequência respiratória, $\mathrm{t}^{\circ} \mathrm{C}$ - temperatura retal. Valores seguidos de letras iguais nas colunas não diferem de forma significativa $(\mathrm{p}>0,05)$.

TABELA 2. Médias e desvios-padrão dos parâmetros bioquímicos séricos, avaliados após uma hora (Momento 0), após seis horas (Momento 1), após doze horas (Momento 2) e após vinte e quatro horas (Momento 3) da administração por via oral de extrato de Kalanchoe blossfeldiana aos cães

\begin{tabular}{cccc}
\hline \multirow{2}{*}{ Momento } & \multicolumn{3}{c}{ Parâmetros } \\
\cline { 2 - 4 } & CK (UI/L) & ALT (UI/L) & FA (U/L) \\
\hline 0 & $42,84^{\mathrm{a}} \pm 27,78$ & $15,69^{\mathrm{a}} \pm 18,34$ & $38.85^{\mathrm{a}} \pm 20.45$ \\
2 & $54,94^{\mathrm{a}} \pm 31,53$ & $19,39^{\mathrm{a}} \pm 25,27$ & $42.00^{\mathrm{a}} \pm 20.23$ \\
3 & $60,95^{\mathrm{a}} \pm 31,48$ & $20,12^{\mathrm{a}} \pm 24,58$ & $36.03^{\mathrm{a}} \pm 16.67$ \\
\hline
\end{tabular}

CK-, ALT-, FA- Valores seguidos de letras iguais nas colunas não diferem de forma significativa $(\mathrm{p}>0,05)$.

TABELA 3. Médias e desvios-padrão dos parâmetros hematológicos, avaliados após uma hora (Momento 0), após seis horas (Momento 1), após doze horas (Momento 2) e após vinte e quatro horas (Momento 3) da administração por via oral de extrato de Kalanchoe blossfeldiana aos cães

\section{Parâmetro}

\begin{tabular}{|c|c|c|c|c|c|c|c|c|}
\hline \multirow{2}{*}{ Momento } & \multirow[b]{2}{*}{$\mathrm{CT}\left(\mathrm{mm}^{3}\right)$} & & \multirow[b]{2}{*}{$\left(\mathrm{mm}^{3}\right)$} & \multirow[b]{2}{*}{$\mathrm{M}\left(\mathrm{mm}^{3}\right)$} & \multirow[b]{2}{*}{$\mathrm{L} \quad\left(\mathrm{mm}^{3}\right)$} & \multirow[b]{2}{*}{$\mathrm{He}\left(\mathrm{mm}^{3}\right)$} & \multirow[b]{2}{*}{$\mathrm{Ht} \quad(\%)$} & \multirow[b]{2}{*}{$\mathrm{F}(\mathrm{mg} \%)$} \\
\hline & & $\left(\mathrm{mm}^{3}\right)$ & & & & & & \\
\hline 0 & $\begin{array}{c}12500^{\mathrm{a}} \pm \\
2617.3\end{array}$ & $\begin{array}{c}8389.3^{\mathrm{ab}} \pm \\
2773.0\end{array}$ & $\begin{array}{c}713.83^{\mathrm{a}} \pm \\
748.34\end{array}$ & $\begin{array}{c}788.33^{\mathrm{a}} \pm \\
325.50\end{array}$ & $\begin{array}{c}2566.8^{\mathrm{a}} \pm \\
1003.4\end{array}$ & $\begin{array}{c}6.03^{\mathrm{a}} \pm \\
0.56\end{array}$ & $\begin{array}{c}44.00^{\mathrm{a}} \pm \\
4.49\end{array}$ & $\begin{array}{l}325^{\mathrm{a}} \pm \\
142.22\end{array}$ \\
\hline 1 & $\begin{array}{c}13350^{\mathrm{a}} \pm \\
2752.3\end{array}$ & $\begin{array}{c}9543.6^{\mathrm{a}} \pm \\
2691.9\end{array}$ & $\begin{array}{c}976.20^{\mathrm{a}} \pm \\
817.53\end{array}$ & $\begin{array}{c}705.10^{\mathrm{a}} \pm \\
312.71\end{array}$ & $\begin{array}{c}2125.2^{\mathrm{a}} \pm \\
843.98\end{array}$ & $\begin{array}{c}5.79^{\mathrm{a}} \pm \\
0.41\end{array}$ & $\begin{array}{c}42.9^{\mathrm{a}} \pm \\
2.42\end{array}$ & $\begin{array}{c}240^{\mathrm{a}} \pm \\
76.27\end{array}$ \\
\hline 2 & $\begin{array}{c}11720^{\mathrm{a}} \pm \\
3785.2\end{array}$ & $\begin{array}{c}9102.3^{\mathrm{ab}} \pm \\
2182.7\end{array}$ & $\begin{array}{l}651^{\mathrm{a}} \pm \\
562.17\end{array}$ & $790^{a} \pm 397.90$ & $\begin{array}{c}2616.7^{\mathrm{a}} \pm \\
927.44\end{array}$ & $\begin{array}{c}6.01^{\mathrm{a}} \pm \\
0.54\end{array}$ & $\begin{array}{c}45.30^{\mathrm{a}} \pm \\
4.02\end{array}$ & $\begin{array}{l}310^{\mathrm{a}} \pm \\
162.09\end{array}$ \\
\hline 3 & $\begin{array}{c}11958^{\mathrm{a}} \pm \\
3053.8\end{array}$ & $\begin{array}{c}7692.3^{\mathrm{b}} \pm \\
2056.1\end{array}$ & $\begin{array}{c}876.42^{\mathrm{a}} \pm \\
887.34\end{array}$ & $\begin{array}{c}822.42^{\mathrm{a}} \pm \\
448.74\end{array}$ & $\begin{array}{c}2087.3^{\mathrm{a}} \pm \\
1019.8\end{array}$ & $\begin{array}{c}5.93^{\mathrm{a}} \pm \\
0.75\end{array}$ & $\begin{array}{c}42.66^{\mathrm{a}} \pm \\
5.53\end{array}$ & $\begin{array}{c}266.67^{\mathrm{a}} \pm \\
98.47\end{array}$ \\
\hline
\end{tabular}

CT- contagem total de leucócitos, N - contagem de neutrófilos, E- contagem de eosinófilos, M- contagem de monócitos, L- contagem de linfócitos, Hecontagem de hemáceas, Ht- hematócrito, F-fibrinogênio. Valores seguidos de letras iguais nas colunas não diferem de forma significativa ( $\mathrm{p}>0,05$ ). 
TABELA 4. Medianas dos escores de eixos cardíacos, médias e desvios-padrão dos parâmetros eletrocardiogáfricos e tipo de arritmia observada, avaliados após uma hora (Momento 0), após seis horas (Momento 1), após doze horas (Momento 2) e após vinte e quatro horas (Momento 3) da administração por via oral de extrato de Kalanchoe blossfeldiana aos cães

\begin{tabular}{ccccccccc}
\hline \multirow{2}{*}{ Momento } & \multicolumn{5}{c}{ Parâmetro } \\
\cline { 2 - 8 } & EEC & DQRS & AQRS & IPR & DOP & AOP & ritmo \\
\hline 0 & $2^{\mathrm{a}}$ & $0.058^{\mathrm{a}} \pm 0.006$ & $1.26^{\mathrm{a}} \pm 0.330$ & $0.093^{\mathrm{a}} \pm 0.023$ & $0.022^{\mathrm{a}} \pm 0.006$ & $0.17^{\mathrm{a}} \pm 0.041$ & AS \\
1 & $2^{\mathrm{a}}$ & $0.056^{\mathrm{a}} \pm 0.008$ & $1.23^{\mathrm{a}} \pm 0.310$ & $0.090^{\mathrm{a}} \pm 0.009$ & $0.020^{\mathrm{a}} \pm 0,001$ & $0.16^{\mathrm{a}} \pm 0.045$ & AS & \\
2 & $2^{\mathrm{a}}$ & $0.056^{\mathrm{a}} \pm 0.008$ & $1.33^{\mathrm{a}} \pm 0.330$ & $0.093^{\mathrm{a}} \pm 0.021$ & $0.022^{\mathrm{a}} \pm 0.006$ & $0.18^{\mathrm{a}} \pm 0.031$ & AS & \\
3 & $2^{\mathrm{a}}$ & $0.060^{\mathrm{a}} \pm 0,003$ & $1.31^{\mathrm{a}} \pm 0.290$ & $0.085^{\mathrm{a}} \pm 0.012$ & $0.020^{\mathrm{a}} \pm 0,001$ & $0.17^{\mathrm{a}} \pm 0.040$ & AS \\
\hline
\end{tabular}

EEC - escore do eixo cardíaco (1-60-90 $\left., 2-30-60^{\circ}, 3-0-30^{\circ}\right)$, DQRS - duração do complexo QRS em milissegundos, AQRS - amplitude do complexo QRS em milivolts, IPR - intervalo entre ondas P e R em segundos, DOP - duração da onda P em milissegundos, AOP - amplitude da onda P em milivolts, AS - arritimia sinusal. Valores seguidos de letras iguais nas colunas não diferem de forma significativa $(\mathrm{p}>0,05)$.

\section{DISCUSSÃO}

O presente trabalho revelou que o extrato de $K$. bloosfeldiana não produziu intoxicações nos cães nas condições em que se desenvolveu este experimento.

MCKENZIE et al. (1987), em experimento com $K$. tubiflorum e K. pinnatum em bovinos, relataram um quadro de intoxicação caracterizado por depressão, incoordenação motora, opistótono, tremores e convulsões. WILLIAMS \& SMITH (1984) observaram um quadro semelhante a este em galinhas.

Com a presença de substâncias cardiotoxicas como os bufanolídeos, comuns na maioria das espécies de Kalanchoe, era esperado que os animais apresentassem sinais de lesões hepáticas, em virtude da metabolização dessas substâncias e, principalmente, lesões cardíacas, com base em sua ação metabólica (STEYN \& HEERDEN, 1998; MILEWSKI \& KHAN, 2006). Isso não foi observado nos animais deste experimento, tendo em vista os resultados dos exames bioquímicos e do eletrocardiograma.

Como os sinais clínicos e os exames realizados não indicaram nenhuma possível lesão causada pela administração da Kalanchoe, optou-se por não sacrificar nenhum animal para a realização de necropsia, o que em contrapartida não permitiu observar eventuais lesões subclínicas, não detectadas pelos exames realizados.

Apesar de a maioria dos relatos e dos trabalhos citarem intoxicação aguda por Kalanchoe (WILLIAMS \& SMITH, 1984; MCKENZIE et al., 1987;
MILEWSKI \& KHAN, 2006), um efeito crônico, cumulativo, não pode ser descartado para esta espécie, sendo necessária a realização de um novo experimento com uma metodologia voltada nesse sentido.

Outra possibilidade é que a Kalanchoe blossfeldiana não seja tóxica como as demais espécies de Kalanchoe, tais como $K$. tubiflorum, $K$. pinnatum, $K$. daigremontianum e K. proliferum, já comprovadas como tóxicas (MACKENZIE et al., 1987).

Os relatos de intoxicação em cães encontrados na literatura não determinam a espécie de Kalanchoe envolvida no quadro, além de ocorrerem especificamente na América do Norte (MILEWSKI \& KHAN, 2006), onde a espécie de Kalanchoe utilizada neste estudo não é a mais comercializada (LORENZI, 2000).

A ampla maioria das plantas da espécie $\mathrm{Ka}$ lanchoe tem sido explorada comercialmente como plantas ornamentais, pelo fato de serem plantas que florescem o ano todo. Esta característica concorreu para a produção em larga escala, sendo, assim, cultivadas artificialmente em estufas. Pode-se especular ainda que, pelas condições artificiais de cultivo dessa planta, ela se diferencie das demais espécies cultivadas em condições naturais, ou seja, é possível que as condições de estufa suprimam ou minimizem um possível efeito tóxico ou deletério ao organismo animal.

A K. blossfeldiana é a espécie de Kalanchoe mais comercializada no Brasil, com mais de uma dezena de variedades (LORENZI, 2000), razão por que motivo foi alvo do presente estudo. Este aspecto vem corroborar a necessidade de definir se realmente 
K. blossfeldiana é toxica ou não para cães, pois a probabilidade de um animal ter contato com este tipo de planta no ambiente doméstico é muito grande.

É interessante pesquisar também os princípios ativos presentes no caule, folha e flor de Kalanchoe blossfeldiana, para que se possa comparar com os princípios ativos encontrados nas demais espécies reconhecidamente tóxicas de Kalanchoe.

\section{CONCLUSÕES}

Os resultados evidenciaram que o extrato de $K$. bloosfeldiana não produz um quadro de intoxicação em cães nas condições em que foi desenvolvido o experimento. Não se notou nenhuma alteração significativa nos parâmetros clínicos dos animais, nem nos valores encontrados nos exames laboratoriais. Tampouco registrou-se indicação de necrose ou isquemia miocárdica por meio do eletrocardiograma.

\section{REFERÊNCIAS}

ANDERSON, L. A.; SCHULTZ, R. A.; JOUBERT, J. P.; PROZESKY, L. Krimpsiekte and acute cardiac glycoside poisoning in sheep caused by bufadienolides from the plant Kalanchoe laceolata Forsk. Ondeestepoort Journal Veteterinary Research, v. 50, p. 295-300, 1983.

ASHTON, A. R.; POLY, G. M. Adenosine 3' :5'-cyclic monophosphate in higher plants: isolation and characterization of adenosine 3':5'-cyclic monophosphate from Kalanchoe an Agave. The Biochemical Journal, v. 165, p. 27-32, 1977.

GAIND, K. N.; GUPTA, R. L. Flavonoid glycosides from Kalanchoe pinnata. Planta Medica, v. 20, p. 368-373, 1971.

GAIND, K. N.; GUPTA, R. L. Phenolic components from Kalanchoe pinnata. Planta Medica, v. 23, p. 149-153, 1973.

HYAKUTAKE, S.; GROTTA, S. Morphological and anatomical study of Kalanchoe braziliensis cambessaedes (Crassuleceae). Revista de Farmarmacologia e Bioquímica da Universidade São Paulo, v. 10, p. 217-237, 1972.

IBRAHIM, T.; CUNHA, J. M. T.; MADI, K.; da FONSECA, L. M. B.; COSTA, S. S.; KOATZ, G. Immunomodulatory and antiinflammatory effects of Kalanchoe brasiliensis. International Immunopharmacology, v. 2, p. 875-883, 2002.

LIPETZ, J. Wound healing in Kalanchoe. Annals of the New York Academy of Sciences, v. 144, p. 320-325, 1967.
LORENZI, H. Plantas daninhas do Brasil. São Paulo: Instituto Plantarum, 2000. $650 \mathrm{p}$.

MASVINGWE, C.; MAVENYENGWA, M. Kalanchoe lanceolata poisoning in Brahman cattle in Zimbabwe: the first field outbreak. Journal of the South African Veterinary Association, v. 68, p. 18-20, 1997.

McCURNIN, D. M.; POFFENBARGER, E. M. Small animal physical diagnosis and clinical procedures. Philadelphia: W. B. Saunders, 1991. 222 p.

McKENZIE, R. A.; FRANKE, F. P.; DUNSTER, P. J. The toxicity to cattle and bufadienolide content of six Bryophillum species. Australian Veterinary Journal, v. 64, p. 298-301, 1987.

McKENZIE, R. A.; DUNSTER, P. J. Hearts and flowers: Bryophyllum poisoning of cattle. Australian Veterinary Journal, v. 63, p. 222-227, 1986.

MILEWSKI, L. M.; KHAN, S. A. An overview of potentially life-threatening poisonous plants in dogs and cats. Journal of Veterinary Emergency and Critical Care, v. 16, n. 1, p. 25-33, 2006.

MOURÃO, R. H.; SANTOS, F. O.; FRANZOTTI, E. M.; MORENO, M. P. Antiinflammatory activity and acute toxicity $\left(\mathrm{LD}_{50}\right)$ of the juice of Kalanchoe brasiliensis (Comb.) leaves picked before and during blooming. Phytotherapy Research, v. 13, p. 352-354, 1999.

PEREZ, A. C.; CORREA, M. F.; BORGES, S. R. Anti-ulcer activity from the leaves of Bryophyllum $s p$. Archives of Veterinary Science, v. 4, n. 1, p. 111-112, 1999.

SALAHDEEN, H. M.; YEMITAN, O. K. Neuropharmacological effects of aqueous leaf extract of Bryophyllum pinnatum in mice. African Journal of Biomedical Research, v. 9, p. 101-107, 2006.

SILVA, S. A.; COSTA, S. S.; MENDONÇA, S. C.; SILVA, E. M. Therapeutic effect of oral Kalanchoe pinnata leaf extract in murine leishmaniasis. Acta Tropical, v. 60, p. 201-210, 1995.

SILVA, S. A.; COSTA, S. S.; ROSSI-BERGMANN, B. The anti-leishmanial effect of Kalanchoe is mediated by nitric oxide intermediates. Parasitology, v. 118, p. 575-582, 1999.

STEYN, P. S.; HEERDEN, F. R. Bufanolides of plant and animal origen. Natural Product Reports, v. 15, p. 397- 413, 1998.

UMBUZEIRO-VALENT, G.; ROUBICEK, D. A.; HAEBISCH, A. B. Mutagenic and antimutagenic evaluation of the juice of the leaves of Bryophyllum calycinum (Kalanchoe pinnata), a plant 
with antihistamine activity. Environmental and Molecular Mutagenesis, v. 33, p. 325-327, 1999.

VARMA, R. K.; GARG, B. D.; AHMAD, A. Pharmacodynamic and toxicological studies of Kalanchoe integra stem. Materia Medica Polona, v. 18, p. 207-210, 1986.
WAGNER, H.; FISCHER, M.; LOTTER, H. Isolation and structure determination of daigremontianin, a novel bufadienolide from Kalanchoe daigremontiana. Planta Medica, v. 2, p. 169-170, 1985.

WILLIAMS, M. C.; SMITH, M. C. Toxicity of Kalanchoe spp to chicks. American Journal Veterinary Research, v. 45, p. 543-546, 1984.

Protocolado em: 16 out. 2007. Aceito em: 29 jul. 2010. 